\title{
ON THE LOCAL SPECTRA OF SEMINORMAL OPERATORS ${ }^{1}$
}

\author{
KEVIN F. CLANCEY
}

\begin{abstract}
Two theorems on the local spectra of seminormal operators are deduced. In the first theorem it is shown that when $T$ is hyponormal any solution of the equation $(T-\lambda) x(\lambda)=x$ on an open set in the plane is necessarily analytic. The second theorem establishes the existence of vectors with small local spectra for a cohyponormal operator with a finite rank self-commutator.
\end{abstract}

Let $T$ be an operator on a Hilbert space $\mathcal{H}$. For $x$ a vector in $\mathcal{H}$ the local resolvent $(T-\lambda)^{-1} x$ is analytic on the resolvent set $\rho(T)$. In case any two analytic extensions of $(T-\lambda)^{-1} x$ agree on their common domain, then the operator $T$ is said to have the single valued extension property. When $T$ has the single valued extension property the domain of the maximal analytic continuation $\tilde{x}$ of the local resolvent is denoted by $\rho_{T}(x)$. The complementary set $\sigma_{T}(x)=\mathrm{C} \backslash \rho_{T}(x)$ is called the local spectrum of $x$. If $T$ has the single valued extension property, then for $\delta$ a closed subset in the plane $X_{T}(\delta)$ will denote the linear manifold consisting of vectors $x$ such that $\sigma_{T}(x)$ is contained in $\delta$.

An operator $S$ on $\mathcal{H}$ is called seminormal in case its selfadjoint selfcommutator $D=S^{*} S-S S^{*}$ is semidefinite. In case $D \geqslant 0$, the operator $S$ is called hyponormal and when $D \leqslant 0$, the operator $S$ is called cohyponormal. There is a marked difference between the local spectral theories for hyponormal and cohyponormal operators. It is known that if $H$ is hyponormal, then $H$ has the single valued extension property. Further $X_{H}(\delta)$ is closed for all closed $\delta \subset \mathbf{C}$. This latter result appears in Stampfli [7] with the hypothesis that the spectrum $\sigma(H)$ consists of continuous spectrum. Radjabalipour [6] has shown that the latter hypothesis on the spectrum of $H$ is unnecessary. It is apparently unknown (excepting trivial cases where $H^{*}$ has eigenvalues) whether $\delta$ can be chosen so that $X_{H}(\delta)$ provides a nontrivial invariant subspace for the operator $H$. On the other hand when $C$ is a nonnormal cohyponormal operator with the single valued extension property there are nonzero vectors with local spectra a proper subset of $\sigma(C)$. Whether $X_{C}(\delta)$ is closed for closed $\delta \subset \mathbf{C}$ is not known. These vectors with small local spectra are provided by a result of Putnam [5] which asserts that any vector in the range of $C C^{*}-C^{*} C$ belongs to the range of $C-\lambda$, for all $\lambda \in \mathbf{C}$. As we shall see below there are no nonzero vectors in the range of $H-\lambda$, for all

Received by the editors October 31, 1977 and, in revised form, March 28, 1978.

AMS (MOS) subject classifications (1970). Primary 47B20.

${ }^{1}$ This work was supported in part by a grant from the National Science Foundation. 
$\lambda \in \mathbf{C}$, when $H$ is a hyponormal operator.

Let $T$ be an operator on $\mathcal{H}$ and $\delta \subset \mathbf{C}$ closed. The notation

$$
Z_{T}(\delta)=\bigcap_{\lambda \notin \delta}(T-\lambda) \mathcal{H}
$$

will be employed.

THEOREM 1. Let $H$ be a hyponormal operator on $\mathcal{H}$ and $\delta \subset \mathrm{C}$ closed. Then $Z_{H}(\delta)=X_{H}(\delta)$.

The above result answers a question of Radjabalipour [6]. Theorem 1 improves a result of Stampfli and Wadhwa [8]. The possibility that $H$ be a normal operator is not ruled out. For the case where $H$ is normal the result in Theorem 1 appears in Putnam [6] and implicitly in [2].

An immediate corollary is the following.

COROllary. Let $H$ be hyponormal. Then $Z_{H}(\phi)=(0)$.

Although in the nonnormal cohyponormal case it is possible to find vectors with local spectra a proper subset of the spectrum, it is not easy to control the location of the local spectra. In this direction we establish:

THEOREM 2. Let $C$ be a cohyponormal operator on $\mathcal{H}$ possessing the single valued extension property and having a finite rank self-commutator. Let $\Delta$ be an open disc such that $\Delta \cap \sigma(C) \neq \varnothing$. There exists a vector $x$ in $\mathcal{H}$ such that $\sigma_{C}(x) \subset \Delta$.

1. Preliminaries to Theorem 1. In this section we record lemmas needed for the proof of Theorem 1 .

LEMMA 1. Let $T$ be an operator without point spectra and $x \in \mathcal{H}$. Let $\Delta_{x}$ be the set of $\lambda$ in $\mathbf{C}$ for which there is a vector $x(\lambda)$ satisfying $(T-\lambda) x(\lambda)=x$. Then $\|x(\lambda)\|$ is a lower semicontinuous function on $\Delta_{x}$.

Proof. Write $T-\lambda$ as $T_{\lambda}$. Let $T_{\lambda}^{*}=U_{\lambda} P_{\lambda}$ be the polar factorization of $T_{\lambda}^{*}$. The operator $U_{\lambda}$ is coisometric with null space the same as $T_{\lambda}^{*}$ and $P_{\lambda}=$ $\left(T_{\lambda} T_{\lambda}^{*}\right)^{1 / 2}$. Let $P_{\lambda}=\int t d E^{\lambda}(t)$ denote the spectral resolution of $P_{\lambda}$. It is easy to verify that if $(T-\lambda) x(\lambda)=x$, then

$$
\|x(\lambda)\|^{2}=\int t^{-2} d\left\|E^{\lambda}(t) x\right\|^{2}
$$

This last identity and in fact the lower semicontinuity of $\|x(\lambda)\|$ follow from the equalities

$$
\int t^{-2} d\left\|E^{\lambda}(t) x\right\|^{2}=\lim _{\mu \rightarrow 0^{+}} \int(t+\mu)^{-2} d\left\|E^{\lambda}(t) x\right\|^{2}=\lim _{\mu \rightarrow 0^{+}}\left(\left(P_{\lambda}+\mu\right)^{-2} x, x\right) .
$$

The lower semicontinuity is implied by the continuity of $\left(\left(P_{\lambda}+\mu\right)^{-2} x, x\right)$ as a function of $\lambda$, when $\mu>0$ is fixed. This ends the proof.

The following lemma appears in Stampfli [7, Theorem 1]. Actually as stated 
in Stampfli one requires the spectrum of $T$ to consist of continuous spectrum. This hypothesis is removed in Radjabalipour [6].

Lemma 2. Let $H$ be hyponormal without eigenvalues and let $x \in \mathcal{H}$. Then

$$
\|\tilde{x}(\lambda)\| \leqslant \frac{\|x\|}{\operatorname{dist}\left(\lambda, \sigma_{H}(x)\right)} .
$$

The final lemma which we need is stated in Radjabalipour [6, Theorem 1] (see, also Stampfli and Wadhwa [8]).

LEMMA 3. Let $H$ be a hyponormal operator without eigenvalues and $\delta \subset \mathrm{C}$ be closed. Suppose there is a bounded function $x(\lambda)$ satisfying $(H-\lambda) x(\lambda)=x$, $\lambda \notin \delta$. Then $x(\lambda)$ is analytic on $\mathrm{C} \backslash \delta$.

2. Proof of Theorem 1. In the proof of the theorem it can be assumed that the operator $H$ has no eigenvalues. Let $\Delta$ be a closed disc. Assume that $x \in H$ satisfies $x \in \bigcap_{\lambda \in \Delta}(H-\lambda) \mathcal{H}$. For $\lambda \in \Delta$, the vector $x(\lambda)$ will be assumed to satisfy $(H-\lambda) x(\lambda)=x$. It will be shown that the interior $\Delta^{\circ}$ of $\Delta$ is contained in $\rho_{H}(x)$. The theorem follows easily from this last statement.

Suppose to the contrary that $\Delta^{\circ} \cap \sigma_{H}(x) \neq \varnothing$.

For $n=1,2, \ldots$, set $F_{n}=\left\{\lambda \in \Delta \cap \sigma_{H}(x):\|x(\lambda)\| \leqslant n\right\}$. It follows from the lower semicontinuity of $\|x(\lambda)\|$ (Lemma 1) that $F_{n}$ is closed and by hypothesis $\cup_{n=1}^{\infty} F_{n}=\Delta \cap \sigma_{H}(x)$. The Baire Category Theorem implies that for some $m$ the set $F_{m} \cap \Delta^{\circ}$ has interior in the relative topology on $\Delta \cap$ $\sigma_{H}(x)$. This means there is an open disc $D \subset \Delta^{\circ}$ with center in $\sigma_{H}(x)$ so that $\|x(\lambda)\|<m$, for all $\lambda \in D \cap \sigma_{H}(x)$. Let $D^{\prime}$ be the disc with same center as $D$ and radius equal to one-half the radius of $D$. Let $\lambda_{0} \in D^{\prime} \cap \rho_{H}(x)$ (if such a $\lambda_{0}$ exists) and choose $\gamma_{0}$ in $D \cap \sigma_{H}(x)$ such that $\left|\lambda_{0}-\gamma_{0}\right|=\operatorname{dist}\left(\lambda_{0}, \sigma_{H}(x)\right)$. Set $z_{0}=x\left(\gamma_{0}\right)=\left(H-\gamma_{0}\right)^{-1} x$. It is easy to see that $\sigma_{H}(x)=\sigma_{H}\left(z_{0}\right)$ and for $\lambda \in \rho_{H}(x)$

$$
z(\lambda)=\frac{x(\lambda)-x\left(\gamma_{0}\right)}{\lambda-\gamma_{0}}
$$

satisfies $(H-\lambda) z(\lambda)=z_{0}$. It follows from Lemma 2 that

$$
\left\|z\left(\lambda_{0}\right)\right\|=\frac{\left\|x\left(\lambda_{0}\right)-z_{0}\right\|}{\left|\lambda_{0}-\gamma_{0}\right|} \leqslant \frac{\left\|z_{0}\right\|}{\operatorname{dist}\left(\lambda_{0}, \sigma_{H}\left(z_{0}\right)\right)} .
$$

Since $\operatorname{dist}\left(\lambda_{0}, \sigma_{H}\left(z_{0}\right)\right)=\operatorname{dist}\left(\lambda_{0}, \sigma_{H}(x)\right)=\left|\lambda_{0}-\gamma_{0}\right|$, we have $\left\|x\left(\lambda_{0}\right)\right\| \leqslant 2\left\|z_{0}\right\|$ $<2 m$. In any case $\|x(\lambda)\|<2 m$, for all $\lambda \in D^{\prime}$, and from Lemma 3 we conclude that $x(\lambda)$ is analytic on $D^{\prime}$. This contradicts the assumption that the center of $D^{\prime}$ belongs to $\sigma_{H}(x)$ and ends the proof.

3. Proof of Theorem 2. Let $C$ be a cohyponormal operator with the single valued extension property. It will be assumed that the self-commutator of $C$ has finite rank $N$ and we will write $D=C C^{*}-C^{*} C=\sum_{i=1}^{N}\left(, \psi_{i}\right) \psi_{i}$ where $\psi_{i}$ 
is orthogonal to $\psi_{j}$ for $i \neq j$. Let $\pi_{0}(C)$ denote the collection of eigenvalues of C. Putnam [5] has shown that there are weakly continuous functions $\tilde{\psi}_{i}$ : C $\backslash \pi_{0}(C) \rightarrow \mathcal{K}$ such that $(C-z) \tilde{\psi}_{i}(z)=\psi_{i},\left\|\tilde{\psi}_{i}(z)\right\| \leqslant 1, i=1, \ldots, N, z \in$ $\mathrm{C} \backslash \pi_{0}(C)$. Below it will be shown that if $\Delta$ is a disc with $\Delta \cap\left[\sigma(C) \backslash \pi_{0}(C)\right]$ $\neq \varnothing$, then one of the functions $\tilde{\psi}_{1}, \ldots, \tilde{\psi}_{N}$ fails to be analytic on $\Delta$.

In the remainder of this section it will be assumed that the operator $C$ is completely nonnormal. This means that there are no nontrivial reducing subspaces of $\mathcal{H}$ on which $C$ is a normal operator. This ensures (among other things) that $\pi_{0}\left(C^{*}\right)=\varnothing$. Let $z \in \sigma(C) \backslash \pi_{0}(C)$ and let $C-z=W_{z} P_{z}^{1 / 2}$ be the polar factorization of $C-z$; here, $P_{z}=(C-z)^{*}(C-z)$ and $W_{z}$ is unitary. It is easy to verify that $W_{z} P_{z}=\left(P_{z}+D\right) W_{z}$.

A result of Krein [3] asserts that existence of a measurable function $\delta_{z}$ with compact support in $[0, \infty)$ satisfying $0 \leqslant \delta_{z} \leqslant N$ such that

$$
\operatorname{det}\left[I_{N}-\left[\left(\left(P_{z}+D-\lambda\right)^{-1} \psi_{i}, \psi_{j}\right)\right]_{N \times N}\right]=\exp \left\{-\int_{0}^{\infty} \frac{\delta_{z}(t)}{t-\lambda} d t\right\}
$$

for all $\lambda$ such that $\operatorname{Re} \lambda \notin[0, \infty)$. In the preceding equation $I_{N}$ denotes the $N \times N$ identity matrix and $\left[\left(\left(P_{z}+D-\lambda\right)^{-1} \psi_{i}, \psi_{j}\right)\right]_{N \times N}$ is the $N \times N$ matrix with $i j$ entry $\left(\left(P_{z}+D-\lambda\right)^{-1} \psi_{i}, \psi_{j}\right)$.

We would like to take the limit in equation (1) as $\lambda \rightarrow 0^{-}$. Our argument proceeds as in Putnam [5]. One notes for $k=1, \ldots, N$ and $\lambda<0$

$$
\begin{aligned}
\left\|\left(P_{z}+D-\lambda\right)^{-1 / 2} \psi_{k}\right\|^{2} & =\left(\left(P_{z}+D-\lambda\right)^{-1} \psi_{k}, \psi_{k}\right) \\
& \leqslant\left((D-\lambda)^{-1} \psi_{k}, \psi_{k}\right)=\frac{\left\|\psi_{k}\right\|^{2}}{\left\|\psi_{k}\right\|^{2}-\lambda} \leqslant 1 .
\end{aligned}
$$

It follows that $\psi_{k}$ belongs to the domain of $\left(P_{z}+D\right)^{-1 / 2}$ and

$$
\lim _{\lambda \rightarrow 0^{-}}\left(P_{z}+D-\lambda\right)^{-1 / 2} \psi_{k}=\left(P_{z}+D\right)^{-1 / 2} \psi_{k}, \quad k=1, \ldots, N .
$$

Taking the limit as $\lambda \rightarrow 0^{-}$in equation (1) one obtains

$\operatorname{det}\left[I_{N}-\left[\left(\left(P_{z}+D\right)^{-1 / 2} \psi_{i},\left(P_{z}+D\right)^{-1 / 2} \psi_{j}\right)\right]_{N \times N}\right]=\exp -\int_{0}^{\infty} \frac{\delta_{z}(t)}{t} d t$

Using the facts that $W_{z} W_{z}^{*}=I$ and $W_{z}^{*}\left(P_{z}+D\right)^{-1 / 2} \psi_{k}=\tilde{\psi}_{k}(z)$ we can write

$$
\operatorname{det}\left[I_{N}-\left[\left(\tilde{\psi}_{i}(z), \tilde{\psi}_{j}(z)\right)\right]_{N \times N}\right]=\exp \left[-\int_{0}^{\infty} \frac{\delta_{z}(t)}{t} d t\right] .
$$

Carey and Pincus [1] have identified the function $\delta_{z}$ in the following manner. There exists an integrable function $G_{z}^{P}$ on the cylinder $[0, \infty) \times T$ such that 


$$
\begin{aligned}
\operatorname{det}\left[I_{N}+\right. & \left.\left(W_{z}\left(P_{z}+D-\lambda\right)^{-1}\left(W_{z}-\tau\right)^{-1} \psi_{i}, \psi_{j}\right)_{N \times N}\right] \\
& =\exp \frac{1}{2 \pi i} \int_{0}^{\infty} \int_{0}^{2 \pi} G_{z}^{P}\left(t, e^{i \theta}\right) \frac{d e^{i \theta}}{e^{i \theta}-\tau} \frac{d t}{t-\lambda}
\end{aligned}
$$

for all $\lambda \notin[0, \infty)$ and $|\tau| \neq 1$. The identity

$$
\delta_{z}(t)=\frac{1}{2 \pi} \int_{0}^{2 \pi} G_{z}^{P}\left(t, e^{i \theta}\right) d \theta \text { a.e. }
$$

is established in [1]. The function $G_{z}^{P}$ is referred to as the polar principal function for the operator $C_{z}$. There is a second principal function $G$ defined for the operator $C$. Write $C=U-i V$ where $U, V$ are selfadjoint. There exists [1] an integrable function $G$ on $R^{2}$ such that

$$
\begin{aligned}
\operatorname{det}\left[I_{N}+\right. & {\left.\left[\left((V-v)^{-1}(U-u)^{-1} \psi_{i}, \psi_{j}\right)\right]_{N \times N}\right] } \\
& =\exp \iint G(\nu, \mu) \frac{d \nu}{\nu-v} \frac{d \mu}{\mu-u}
\end{aligned}
$$

The basic result relating $G$ and $G_{z}^{P}$ is the identity

$$
G_{z}^{P}\left(t, e^{i \theta}\right)=G(\nu, \mu) \text { a.e. }
$$

where $\mu+i \nu-\bar{z}=\sqrt{t} e^{i \theta}[1]$.

Then

$$
\begin{aligned}
\int_{0}^{\infty} \frac{\delta_{z}(t)}{t} d t & =\frac{1}{2 \pi} \int_{0}^{\infty} \int_{0}^{2 \pi} \frac{G_{z}^{P}\left(t, e^{i \theta}\right)}{t} d t d \theta \\
& =\frac{1}{\pi} \iint \frac{G(\nu, \mu)}{|\mu+i \nu-\bar{z}|^{2}} d \mu d \nu
\end{aligned}
$$

There is a subset $B \subset A=\{z=\mu+i \nu: G(\nu, \mu) \neq 0\}$ such that the last integral is infinite at every $\bar{z} \in B$, moreover the planar measure of $A \backslash B$ is zero.

It is known that the essential closure of $A$ is $\sigma\left(C^{*}\right)$. This implies the essential closure of $B$ is $\sigma\left(C^{*}\right)$. Thus whenever $\Delta$ is a disc intersecting $\sigma(C)$ there are points in $\Delta$ at which $\int_{0}^{\infty}\left(\delta_{z}(t) / t\right) d t=\infty$. Consequently, if $\Delta$ is a disc intersecting $\sigma(C) \backslash \pi_{0}(C)$ there are points $z$ in $\Delta$ with

$$
\operatorname{det}\left[I_{N}-\left[\left(\tilde{\psi}_{i}(z), \tilde{\psi}_{j}(z)\right)\right]_{N \times N}\right]=0
$$

It will be shown that the matrix $R(z)=\left[\left(\tilde{\psi}_{i}(z), \tilde{\psi}_{j}(z)\right)\right]$ satisfies $0<R(z)<$ $I_{N}$. Note first

$$
\tilde{\psi}_{i}(z)=W_{z}^{*}\left(P_{z}+D\right)^{-1 / 2} \psi_{i} \quad(1 \leqslant i<N)
$$

Therefore 


$$
\begin{aligned}
\left(\tilde{\psi}_{i}(z), \tilde{\psi}_{j}(z)\right) & =\left(\left(P_{z}+D\right)^{-1 / 2} \psi_{i},\left(P_{z}+D\right)^{-1 / 2} \psi_{j}\right) \\
& =\lim _{\lambda \rightarrow 0}\left(\left(P_{z}+D-\lambda\right)^{-1 / 2} \psi_{i},\left(P_{z}+D-\lambda\right)^{-1 / 2} \psi_{j}\right) \\
& =\lim _{\lambda \rightarrow 0}\left(\left(P_{z}+D-\lambda\right)^{-1} \psi_{i}, \psi_{j}\right) .
\end{aligned}
$$

The result will follow when it is established that

$$
R(z, \lambda)=\left[\left(\left(P_{z}+D-\lambda\right)^{-1} \psi_{i}, \psi_{j}\right)\right]<I_{N}
$$

Let $x=\left[x_{1}, \ldots, x_{N}\right] \in C^{N}$. Then

$$
\langle R(z, \lambda) x, x\rangle=\left(\left(P_{z}+D-\lambda\right)^{-1} \omega, \omega\right)
$$

where $\omega=\sum_{i=1}^{N} \bar{x}_{i} \psi_{i}$. Since $\left(P_{z}+D-\lambda\right)^{-1} \leqslant(D-\lambda)^{-1}$ we have

$$
\langle R(z, \lambda) x, x\rangle \leqslant\left((D-\lambda)^{-1} \omega, \omega\right) .
$$

Using the fact that $D=\sum_{i=1}^{N} \psi_{i} \otimes \psi_{i}$ with $\psi_{i}$ orthogonal one computes

$$
(D-\lambda)^{-1} \omega=\sum_{i=1}^{N} \frac{\bar{x}_{i}}{\left\|\psi_{i}\right\|^{2}-\lambda} \psi_{i}
$$

Therefore

$$
\langle R(z, \lambda) x, x\rangle \leqslant \sum_{i=1}^{N} \frac{\left|x_{i}\right|^{2}\left\|\psi_{i}\right\|^{2}}{\left\|\psi_{i}\right\|^{2}-\lambda}<\sum_{i=1}^{N}\left|x_{i}\right|^{2} .
$$

This shows $R(z, \lambda) \leqslant I_{N}$ and the estimate $R(z) \leqslant I_{N}$ follows.

Note that if $e_{1}, \ldots, e_{N}$ are orthonormal in $\mathcal{H}$, then with $A(z)=$ $\sum_{i=1}^{N}\left(, e_{i}\right) \tilde{\psi}_{i}(z)$, the matrix $R(z)$ is (unitarily equivalent to) the product $A^{*}(z) A(z)$.

Suppose now that $\Delta \cap\left[\sigma(C) \backslash \pi_{0}(C)\right] \neq \varnothing$ and $\tilde{\psi}_{1}, \ldots, \tilde{\psi}_{N}$ are analytic on $\Delta$. Then from (5) it follows that the norm of $A(z)$ is achieved at $z_{0} \in \Delta$. This means for some nonzero $f$,

$$
\left\|A\left(z_{0}\right) f\right\|=1=\max _{z \in \Delta}\|A(z)\| .
$$

It follows easily from the maximum modulus theorem that $A(z) f$ is constant on $\Delta$.

This yields $\sum_{i=1}^{N}\left(f, e_{i}\right) \tilde{\psi}_{i}(z)$ is a constant vector $g$ on $\Delta$. Let $h=$ $\sum_{i=1}^{N}\left(f, e_{i}\right) \psi_{i}$. Then $(C-z) g=h(z \in \Delta)$ which is impossible. We conclude that one of $\tilde{\psi}_{1}, \ldots, \tilde{\psi}_{N}$ is not analytic on $\Delta$.

The proof of Theorem 2 can now be completed as follows. Let $\Delta \cap \sigma(C)$ $\neq \varnothing$. If $\lambda \in \Delta \cap \pi_{0}(C)$, then any eigenvector $f_{\lambda}$ associated with $\lambda$ satisfies $\sigma_{C}\left(f_{\lambda}\right)=\{\lambda\} \subset \Delta$. If no such $\lambda$ exists then one of the weakly continuous functions $\tilde{\psi}_{1}, \ldots, \tilde{\psi}_{N}$ say $\tilde{\psi}_{p}$ fails to be analytic on $\Delta$. This means that for some simple closed curve $\Gamma \subset \Delta, \varphi=\int_{\Gamma} \tilde{\psi}_{p}(z) d z \neq 0$. As in Stampfli [7] one shows $\sigma_{C}(\varphi) \subset \Delta$. This ends the proof of Theorem 2 . 


\section{REFERENCES}

1. R. W. Carey and J. D. Pincus, Mosaics, principal functions, and mean motion in von Neumann algebras, Acta. Math. 138 (1977), 151-216.

2. B. E. Johnson, Continuity of linear operators commuting with continuous linear operators, Trans. Amer. Math. Soc. 128 (1967), 88-102.

3. M. G. Krein, Perturbation determinants and a formula for the traces of unitary and self-adjoint operators, Dokl. Akad. Nauk SSSR 144 (1962), 268-271.

4. C. R. Putnam, Ranges of normal and subnormal operators, Michigan Math. J. 18 (1971), 33-36.

5.

, Hyponormal contractions and strong power convergence, Pacific J. Math. 57 (1975), 531-538.

6. M. Radjabalipour, Ranges of hyponormal operators, Illinois J. Math. 21 (1977), 70-75.

7. J. G. Stampfli, A local spectral theory for operators. V: Spectral subspaces for hyponormal operators, Trans. Amer. Math. Soc. 217 (1976), 285-296.

8. J. G. Stampfli and B. L. Wadhwa, On dominant operators, Monatsh. Math. 84 (1977), 143-153.

Department of Mathematics, University of Georgia, Athens, Grorgin 30602 